This is a preprint version of the article published in Micro and Nanofluidics with DOI 10.1007/s10404-014-1365-3. For the final published version, please visit http://link.springer.com/article/10.1007\%2Fs10404-014-1365-3.

\title{
Title: Capillary pumps with constant flow rate
}

Author: Wouter van der Wijngaart

Affiliation: KTH Royal Institute of Technology, Micro and Nanosystems Department, Stockholm, Sweden

Contact information:

Address: Micro and Nanosystems, KTH Royal Institute of Technology, Osquldas väg 10, 10044 Stockholm, Sweden

Email: wouter@kth.se

\section{Abstract}

This paper unambiguously derives, ab initio starting from the Navier-Stokes and Laplace equations, the geometric parameters defining capillary pumps with rectangular crosssection with constant volumetric flow rate and steady velocity profile. The parametric formulation of the channel shape is derived using Taylor series approximations of the capillary pressure and the hydrodynamic flow resistance with negligible error. First, the design parameters are derived for a capillary pump consisting of a single channel and illustrated with an example. Thereafter, the design parameters for multichannel and for micropillar array capillary pumps are derived. Finally, the design of capillary pumps for multistep constant flow rates derived and those for arbitrarily varying flow rates are discussed. 


\section{Introduction}

Capillary systems are widely used for manipulating liquids in heat pipes, regulating fluid-flow in low-gravity environment in space, patterning biomolecules on surfaces, immunoassays, and diagnostic applications. CPs are notably used in diagnostic assays to flow accurate volumes of sample (i.e. 1-10 $\mu$ l) over an upstream assay surface to reach a high sensitivity. The volume of the CP serves as a metering device - the pump's capacity sets the volume of the liquid drawn in - and as a waste reservoir for reagents. For immunoassays, it is often preferred to keep the velocity field $\vec{u}$ steady at an optimal state during the incubation step. The latter is achieved if the pump volumetric flow $Q$ is constant:

$\mathrm{Q}(\mathrm{t})=\mathrm{Q}_{0}$, with $\frac{d Q}{d t}=0$.

Washburn's equation describes capillary flow in a bundle of parallel cylindrical tubes with constant cross-section as

$x \sim \sqrt{t}$,

and thus

$Q=A \cdot \frac{d x}{d t} \sim t^{-\frac{1}{2}}$,

with $\mathrm{x}$ being the position of the liquid front and A being the flow cross-sectional area. These equations can be extended, with some issues (Chandra, 2009), also to imbibition into porous materials for which both the capillary pressure and the flow resistivity are constant throughout the capillary system. Previous CP design approaches for providing a constant flow are based on providing a constant capillary pressure throughout the CP while not significantly increasing the overall flow resistance as the CP is being filled (Eddowes, 1988). Such designs have been realised in the form of parallel microchannels arranged in an arborescent pattern (Juncker, 2002) or arrays of posts (Gervais, 2011), or meshes (Mirzaei, 2010). Based on a microfluidic design by Melin et al. (Melin, 2004), Safavieh et al. reported a CP design with varying capillary flow based on a meandering channel design containing geometric capillary valves between the meander arms. 


\section{Governing equations}

Table 1. Parameters and variables determining the $\mathrm{CP}$ behaviour.

\begin{tabular}{|c|c|}
\hline Symbol & Description \\
\hline \multicolumn{2}{|r|}{ Geometric parameters } \\
\hline$Q_{0}$ & Desired flow rate \\
\hline$h$ & Channel height \\
\hline$W_{\min }$ & Minimum channel width \\
\hline$L_{c h}$ & The length of a capillary pump, i.e. $\mathrm{L}_{\mathrm{ch}}=\mathrm{s}\left(\mathrm{w}_{\min }\right)$ and $\mathrm{w}\left(\mathrm{L}_{\mathrm{ch}}\right)=\mathrm{w}_{\min }$ \\
\hline$L_{\max }$ & The maximum possible length of a capillary pump, i.e. $L_{\max }=s(0)$ and $w\left(L_{\max }\right)=0$. \\
\hline$R_{0}$ & Hydrodynamic flow resistance of the fluidic network upstream from the CP \\
\hline$l_{e}$ & Hydrodynamic entrance length \\
\hline$w_{\max }$ & Maximum channel width \\
\hline$n$ & Amount of parallel channels in a multichannel and micropillar array design \\
\hline \multicolumn{2}{|r|}{ Liquid properties } \\
\hline$\rho$ & Liquid density \\
\hline$\gamma$ & Liquid surface energy \\
\hline$\mu$ & Liquid viscosity \\
\hline$\theta$ & Liquid-solid contact angle \\
\hline \multicolumn{2}{|r|}{ Variables } \\
\hline$t$ & Time \\
\hline$s$ & Spatial position along the channel centre line. \\
\hline$x$ & Position of the liquid front in the channel \\
\hline$Q(x)$ & Volumetric flow rate when the liquid front is at position $\mathrm{x}$ \\
\hline$\vec{u}$ & Velocity field \\
\hline$\vec{b}$ & Sum of all body forces acting on the liquid (assumed negligibly small) \\
\hline$w(s)$ & Channel width at position s (= inverse function of $\mathrm{s}(\mathrm{w}))$ \\
\hline$s(w)$ & Channel position at which the channel width is $\mathrm{w}(=$ inverse function of $\mathrm{w}(\mathrm{s}))$ \\
\hline$S^{*}$ & Dimensionless position along the channel centre line. \\
\hline$W^{*}$ & Dimensionless channel width \\
\hline$C p$ & Dimensionless number that characterizes the capillary pump shape \\
\hline$P(x)$ & Capillary suction when the liquid front is at position $\mathrm{x}$ \\
\hline$R_{h}(x)$ & Total hydrodynamic flow resistance of the CP with liquid front at position $\mathrm{x}$ \\
\hline$V(x)$ & Volume of the CP between the entrance and position $\mathrm{x}$ \\
\hline$\beta(s)$ & Angle between the channel wall and the channel centre line \\
\hline
\end{tabular}

This work considers a generic capillary pump device that is designed to pump liquid with a constant volumetric flow rate $Q_{0}$ through an up-stream fluidic network for which the total hydrodynamic resistance $\mathrm{R}_{0}$ can be tuned appropriately.

The Navier-Stokes (NS) equation expresses the velocity field of a laminar incompressible flow in a capillary driven system as

$-\nabla P+\mu \nabla^{2} \vec{u}+\vec{b}-\rho \frac{d \vec{u}}{d t}=0$

in which $\vec{u}$ is the velocity field, $\mathrm{P}$ the pressure distribution, $\vec{b}$ the sum of all body forces, and $\rho$ and $\mu$ are the liquid density and viscosity, respectively. In typical lateral flow devices, the body forces $\vec{b}$, such as gravity and electro-fluidic interactions, can be neglected. Considering the a priori demand on a steady velocity field, the fluid acceleration term must be zero everywhere. Let us assume, and below verify, that this demand is fulfilled. The steady flow assumption allows solving the NS equation for any position $\mathrm{x}$ of the liquid front in an expression

$\forall x: Q(x)=\frac{P(x)}{R_{h}(x)}=Q_{0}=\frac{P(s)}{R_{h}(s)}$ 
in which $\mathrm{R}_{\mathrm{h}}(\mathrm{x})$ and $\mathrm{P}(\mathrm{x})$ are the hydrodynamic flow resistance and the pressure drop over the entire liquid plug, respectively.

The pressure of the liquid at the loading port is assumed constant and at atmospheric pressure. Consequently, the pressure drop over the entire liquid plug, $\mathrm{P}(\mathrm{x})$, during capillary filling equals the Laplace pressure at the liquid front.

For increasing positions $\mathrm{s}, \mathrm{R}_{\mathrm{h}}(\mathrm{s})$ increases. Consequently, a constant flow design requires a capillary suction $\mathrm{P}(\mathrm{s})$ that increases with s. CP designs with a constant porosity, and hence constant capillary pressure (Eddowes, 1988; Juncker, 2002; Gervais, 2011; Mirzaei, 2010) therefore inherently feature a flow rate $Q(t)$ that decreases during filling of the CP.

\section{CPs with constant flow rate}

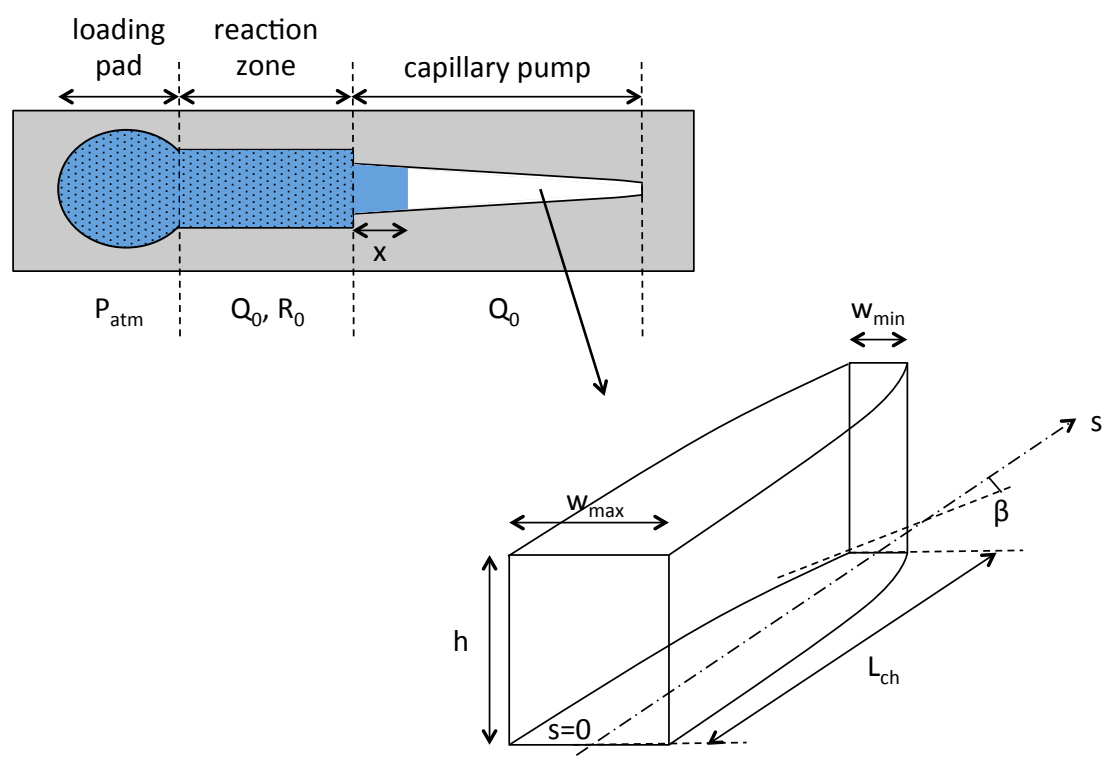

Fig. 1. Schematic top view of a lateral flow test with a single channel CP design, and schematic $3 D$ view of the CP part with constant height $h$ and with the width $w(S)$ decreasing with the channel position $s$.

This section shows how a constant flow CP can be designed in the shape of a single channel with constant height $\mathrm{h}$ and channel width $\mathrm{w}(\mathrm{s})$ monotonously decreasing with $\mathrm{s}$. The maximum channel width occurs at $\mathrm{s}=0$, where $\mathrm{w}(0)=\mathrm{w}_{\max }$ is a design parameter. $\mathrm{A}$ decreasing $w(s)$, in combination with a hydrophylic material surface with equilibrium contact angle $\theta<\frac{\pi}{2}$, ensures an increasing P(s) with increasing values of s.

Eq. 5 will be used to derive the shape w(s) of the channel that leads to a constant flow $\mathrm{CP}$. The hydrodynamic resistance of the wetted device section can be calculated as

$R_{h}(x)=R_{0}+\int_{0}^{x} R(s) \cdot d s$,

with

$R(s)=\frac{d}{d s}\left(\frac{P(s)}{Q(s)}\right)$

being the hydrodynamic resistivity at position s. The hydrodynamic resistivity $\mathrm{R}$ of a channel with rectangular cross section can be expressed in function of its channel width 
$\mathrm{w}$ and height $\mathrm{h}$ and the fluid viscosity $\mu$, as described in any standard literature, e.g. (Bruus, 2008):

$$
\begin{aligned}
& R=\mu \cdot \frac{12}{h \cdot w^{3} \cdot\left[1-\frac{w}{h} \cdot \sum_{n, o d d}^{\infty} \frac{192}{(n \pi)^{5}} \cdot \tanh \left(n \cdot \frac{\pi h}{2 w}\right)\right]} \\
& \approx \mu \cdot \frac{12}{h \cdot w^{3} \cdot\left[1-0.630 \cdot \frac{w}{h}\right]} .
\end{aligned}
$$

Note that the approximation introduced in eq. 9 is valid for $\frac{w}{h}<0.5$. Let us assume, and below verify, that the error stemming from this approximation is negligible. $\mathrm{w} / \mathrm{h} \leq 0.5$ will hereafter be taken as a design requirement.

One can express the capillary suction P(s) as (Man, 1998)

$P(s)=-\frac{d U_{S}}{d V_{l}}=\gamma \cdot\left[\cos (\theta) \frac{d A_{s l}}{d V_{l}}-\frac{d A_{l g}}{d V_{l}}\right]$,

where $U_{s}, V_{l}, A_{s l}, A_{l a}$ and $\gamma$ denote the total surface energy of the channel geometry, the liquid volume in the channel, the total wetted area of the channel, the total liquid-air interfacial surface area and the liquid-gas surface energy, respectively.

To calculate $P(s)$, a zero-th order Taylor series expansion, $w(s)=w(x)$, around $s=x$ can be used where the error induced is negligible (to be verified below). For a zero-th order approximation of $w(s)$, the capillary suction can be written as (see Appendix)

$P(s)=2 \cdot \gamma \cdot \cos (\theta) \cdot\left[\frac{1}{h}+\frac{1}{w}\right]$.

Taking the derivative of eq. 5 allows expressing

$\frac{\mathrm{d} R_{h}(s)}{d s}=\frac{1}{Q_{0}} \cdot \frac{d P(s)}{d s}$.

Substituting

$\frac{\mathrm{d} R_{h}(s)}{d s}=R(s)=\mu \cdot \frac{12}{h \cdot w^{3} \cdot(1-0.63 w / h)}$

and

$\frac{1}{Q_{0}} \cdot \frac{d P(s)}{d s}=\frac{1}{Q_{0}} \cdot \frac{d P(w)}{d w} \cdot \frac{d w}{d s}=\frac{\gamma \cdot \cos (\theta)}{Q_{0}} \cdot \frac{-2}{w^{2}} \cdot \frac{d w}{d s}$

in eq. 12 yields

$\left(0.63 w^{2}-h \cdot w\right) \cdot \frac{d w(s)}{d s}=\frac{6 \cdot \mu \cdot Q_{0}}{\gamma \cdot \cos (\theta)}$.

Integrating both sides over s and rearranging results in:

$s(w)=\frac{\gamma \cdot \cos (\theta)}{6 \cdot \mu \cdot Q_{0}}\left[0.21 w^{3}-\frac{h}{2} \cdot w^{2}-c_{1}\right]$

with $c_{1}$ a constant, determined from the boundary condition $\mathrm{w}=\mathrm{w}_{\max }$ at position $\mathrm{s}=0$ :

$c_{1}=0.21 w_{\max }^{3}-\frac{h}{2} \cdot w_{\max }^{2}$. 
Eq. 16 defines a cubic equation in $\mathrm{w}$, in which $\mathrm{w}$ can be solved as the root for the interval $\left[0, \mathrm{w}_{\max }\right]$, which can be expressed as

$w(s)=\frac{h}{1.26}-\frac{v(s)+v^{*}(s)}{2}+i \cdot \sqrt{3} \frac{v(s)-v^{*}(s)}{2}$,

where $\mathrm{i}$ is the imaginary unit, i.e. $\mathrm{i}^{2}=-1$,

$v(s)=\left[\left(\frac{h}{1.26}\right)^{3}+\frac{c_{1}+\frac{6 . \mu \cdot Q_{0}}{\gamma \cdot \cos (\theta)} s}{0,42}+\sqrt{\left[\left(\frac{h}{1.26}\right)^{3}+\left(\frac{c_{1}+\frac{6 . \mu \cdot Q_{0}}{\gamma \cdot \cos (\theta)}}{0,42}\right)\right]^{2}-\left(\frac{h}{1.26}\right)^{6}}\right]^{1 / 3}$,

and $\mathrm{u}^{*}(\mathrm{~s})$ is the complex conjugate of $\mathrm{u}(\mathrm{s})$. Although $\mathrm{u}(\mathrm{s})$ and its conjugate are complex numbers, the solution for $\mathrm{w}(\mathrm{s})$ is real.

The upstream hydrodynamic resistance $\mathrm{R}_{0}$ must be designed such that

$R_{0}=\frac{P(0)}{Q_{0}}=\frac{2 \gamma \cdot \cos (\theta)}{Q_{0}}\left[\frac{1}{h}+\frac{1}{w_{\max }}\right]$

Taking into account the boundary condition $V(0)=0$, the $C P$ volume $V(s)$ between the $C P$ entrance and a downstream position $\mathrm{s}(\mathrm{w})$ can be calculated as

$V(w)=\int_{w(s)}^{w_{\max }} h \cdot s(w) \cdot d w$

$=\frac{\gamma \cdot \cos (\theta) \cdot h}{6 \cdot \mu \cdot Q_{0}} \cdot\left[\frac{0.21}{4}\left(w_{\max }^{4}-w^{4}\right)-\frac{h}{6} \cdot\left(w_{\max }{ }^{3}-w^{3}\right)-w_{\max }^{2} \cdot\left(0.21 \cdot w_{\max }-\frac{h}{2}\right) \cdot\left(w_{\max }-w\right)\right]$.

The pump design can now be optimised for providing a maximum pump volume. Eq. 21 shows that this requires maximising the values of $h$ and $w_{\max }$, i.e. by choosing $h$ as the maximum manufacturable channel depth $\mathrm{h}_{\max }$ :

$\mathrm{h}=\mathrm{h}_{\max }$

and choosing

$\mathrm{w}_{\max }=\mathrm{h} / 2$.

Such single channel pump can have a maximum channel length

$L_{\max }=s(0)=\frac{0.79 w_{\max }{ }^{3} \cdot \gamma \cdot \cos (\theta)}{48 \cdot \mu \cdot Q_{0}}$.

Inserting Eqs 17 and 23 in Eq. 16 and dividing both sides by Eq. 24 the channel shape can also be written in the dimensionless units $\mathrm{s}^{*}=\mathrm{s} / \mathrm{w}_{\max }$ and $\mathrm{W} * \mathrm{w} / \mathrm{w}_{\max }$ as

$S_{*}=\frac{1}{48 . C p} \cdot\left[0.21 w_{*}^{3}-w_{*}^{2}+0.79\right]$

or

$W_{*}=\frac{1}{2.52}-\frac{v_{+}\left(s_{*}\right)+v_{+}{ }^{*}\left(s_{*}\right)}{4}+i \cdot \sqrt{3} \frac{v_{+}\left(s_{*}\right)-v_{+}{ }^{*}\left(s_{*}\right)}{4}$,

with 
$C p=\frac{\mu \cdot Q_{0}}{w_{\max }^{2} \cdot \gamma \cdot \cos (\theta)}=\frac{\mu \cdot \bar{u}(0)}{\gamma} \cdot \frac{2}{\cos (\theta)}$

being a dimensionless Capillary number at position $s=0$, and

$$
\begin{aligned}
& v_{+}(s)= \\
& {\left[\left(\frac{1}{1.26}\right)^{3}+\frac{0.79}{3.36}\left(\left(\frac{48 . C p}{0.79} \cdot s_{*}\right)^{3}-1\right)+\sqrt{\left[\left(\frac{1}{1.26}\right)^{3}+\frac{0.79}{3.36}\left(\left(\frac{48 . C p}{0.79} \cdot s_{*}\right)^{3}-1\right)\right]^{2}-\left(\frac{1}{1.26}\right)^{6}}\right]^{1 / 3} .}
\end{aligned}
$$

This shape is plotted in Figure 2.

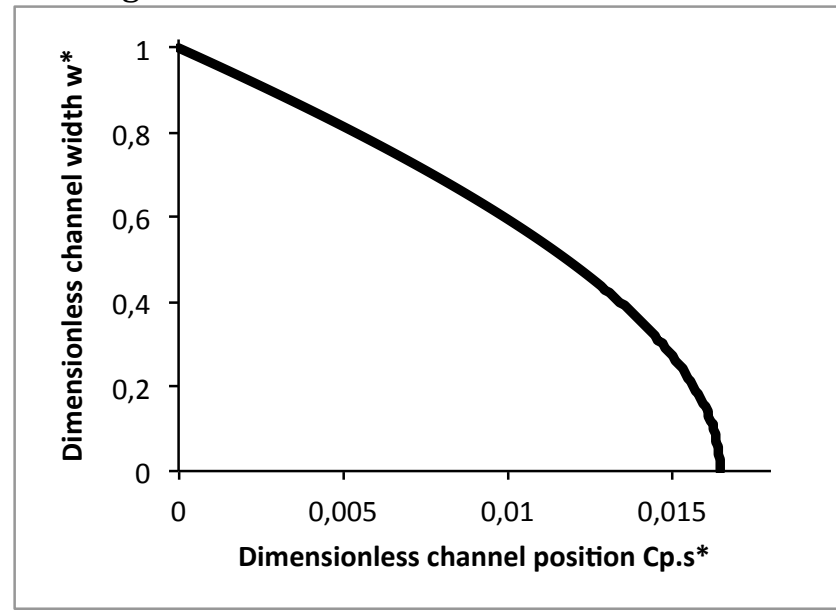

Fig. 2. Shape of the dimensionless channel width $w *$ versus the dimensionless channel position Cp.S*for a capillary channel with constant flow rate.

Eqs. 20, 23, 25 (alternatively 26) and 27 unambiguously describe the shape of a single channel with rectangular cross-section that provides a constant flow rate when used as capillary pump.

For such optimised design, inserting eqs. 23 and 27 in eq. 21 allows expressing

$V_{\text {max }}=V(0)=0.0212 \cdot \frac{h^{3}}{C p}$.

Note that in practical realisations, the channel length may need to be truncated at a position $\mathrm{s}\left(\mathrm{w}_{\min }\right)<\mathrm{L}_{\max }$, in which $\mathrm{w}_{\min }$ is the minimum manufacturable channel width. The above equations are illustrated with an example below.

Table 2. Parameter values used in the example design.

\begin{tabular}{|c|c|}
\hline$Q_{0}$ & $2 \mu \mathrm{l} / \mathrm{min}$ \\
\hline$h$ & $275 \mu \mathrm{m}$ \\
\hline$w_{\min }$ & $20 \mu \mathrm{m}$ \\
\hline$w_{\max }$ & $=\mathrm{h} / 2=137.5 \mu \mathrm{m}$ \\
\hline$\rho$ & $1000 \mathrm{~kg} \cdot \mathrm{m}^{-3}$ \\
\hline$\gamma$ & $0.06 \mathrm{~J} / \mathrm{m}^{2}$ \\
\hline$\mu$ & $8.9 \mathrm{e}-4 \mathrm{~Pa} . \mathrm{s}$ \\
\hline$\theta$ & $\cos (\theta)=0.7$ \\
\hline
\end{tabular}

Figure 3 shows a plot of $w(s), d w / d s$ and $P(x)$, as defined by eqs. 18, 15 and 11, respectively, for a single channel $\mathrm{CP}$ with example geometrical design parameters and 
liquid properties listed in Table 2 . The pump is truncated at position $\mathrm{s}=0.421 \mathrm{~m}$, where $\mathrm{w}(\mathrm{s})=\mathrm{w}_{\min }=20 \mu \mathrm{m}$. The upstream flow resistance required is $\mathrm{R}_{0}=2.75 .10^{13} \mathrm{~Pa} \mathrm{~s} . \mathrm{m}^{-3}$, and the resulting total pump volume $\mathrm{V}_{\max }=10.5 \mu \mathrm{l}$.

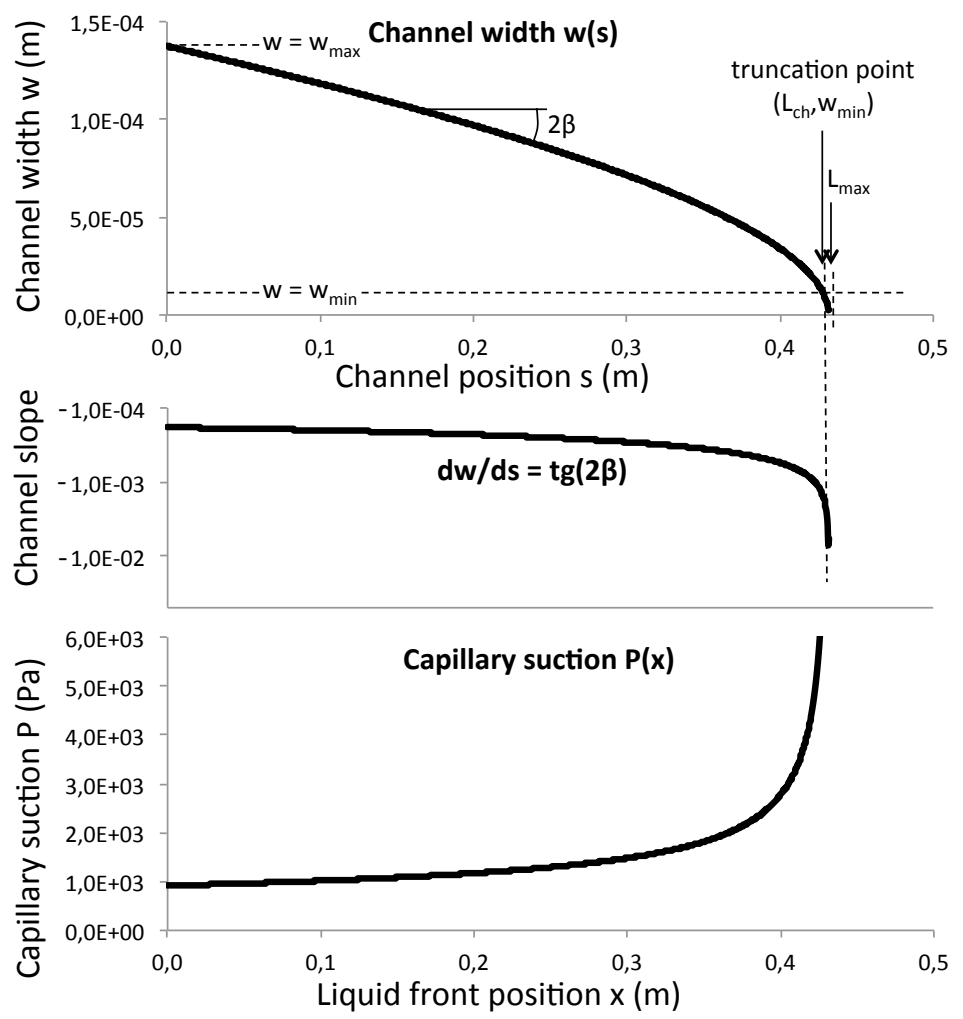

Fig. 3. Plot of the channel width $w(s)$, the channel slope $d w / d s$, and the resulting capillary suction $P(x)$ for a liquid front position $x$ in a single channel $C P$ design using the parametric values listed in Table 2.

The single channel CP described above is relatively long: $\mathrm{L}_{\mathrm{ch}}=\mathrm{s}\left(\mathrm{w}_{\min }\right)=42.1 \mathrm{~cm}$. For footprint efficient implementations, one can consider winding up the channel in a serpentine shape or a spiral shape. The capillary suction in curved channels is the same as in straight channels with the same flow cross-sectional geometry. However, the curvature may locally alter the hydrodynamic flow resistivity as compared to straight channels. One can address such deviations either by an adapted channel shape in curving regions, or by keeping the total volume of the curved regions negligibly small in comparison to the total channel volume.

\section{Verification of the assumptions and approximations}

The above design is based on three assumptions or approximations, and this section will discuss their validity.

The first assumption is that of steady flow. The flow can only be steady if the velocity profile is fully developed. However, there will always exist a region immediately behind the moving liquid front, where the flow is not yet fully developed. The non-steady velocity profile in this region may alter the local hydrodynamic flow resistivity. This change in local resistivity is very difficult to estimate. However, this change can be neglected if both the volume of this region is small compared to the total liquid volume and the difference in resistivity between undeveloped and fully developed laminar flow is limited. The non-steady region has a length that can be estimated being of the same magnitude order as that of the hydrodynamic entrance length, $\mathrm{l}_{\mathrm{e}}$, for continuous flow at 
the entrance of a duct. For laminar flow in a rectangular channel with width $\mathrm{w}$ and height $\mathrm{h}$, and thus hydraulic diameter $D_{h}=\frac{2 h w}{(h+w)}$, the dimensionless hydrodynamic entrance length $0.075<l_{e}{ }^{*}<0.09$, which allows estimating $l_{\mathrm{e}}$ as (Shah, 1978)

$l_{e}=l_{e}^{*} \cdot D_{h} \cdot R e=l_{e}^{*} \cdot \frac{\rho Q_{0}}{\mu} \cdot \frac{4 h w}{(h+w)^{2}} \leq 0.08 \cdot \frac{\rho Q_{0}}{\mu}$.

For typical flow velocities encountered in lateral flow devices, i.e. $Q_{0}<10$ 's of $\mu \mathrm{l} / \mathrm{min}$, the volume of such non-steady region is typically many magnitude orders smaller than that of the total wetted volume, the latter including the entire upstream hydrodynamic resistor $\mathrm{R}_{0}$. For example, for a liquid with water-like density and viscosity, and for a flow rate $\mathrm{Q}_{0}=2 \mu \mathrm{l} / \mathrm{min}, \mathrm{l}_{\mathrm{e}} \leq 2.67 \mu \mathrm{m}$. If we assume the change in resistivity between undeveloped and fully developed laminar flow to be smaller than one magnitude order, the resulting change in the total hydrodynamic resistance $\mathrm{R}_{\mathrm{h}}$ can be neglected.

The second assumption is that $\mathrm{w}(\mathrm{s})$ can be approximated with a zero-th order Taylor series around $\mathrm{x}$ for the derivation of $\mathrm{P}(\mathrm{s})$. The limit on the maximum relative error introduced by this series truncation, $\varepsilon_{P}$, is limited:

$\varepsilon_{P}<\frac{P_{1}(s)-P_{0}(s)}{P_{0}(s)}$

where $\mathrm{P}_{0}(\mathrm{~s})$ and $\mathrm{P}_{1}(\mathrm{~s})$ are the zero-th and first order Taylor approximations, respectively, derived in the Appendix. $\varepsilon_{P}$ is plotted against the slope of w(s) in Fig. 4.

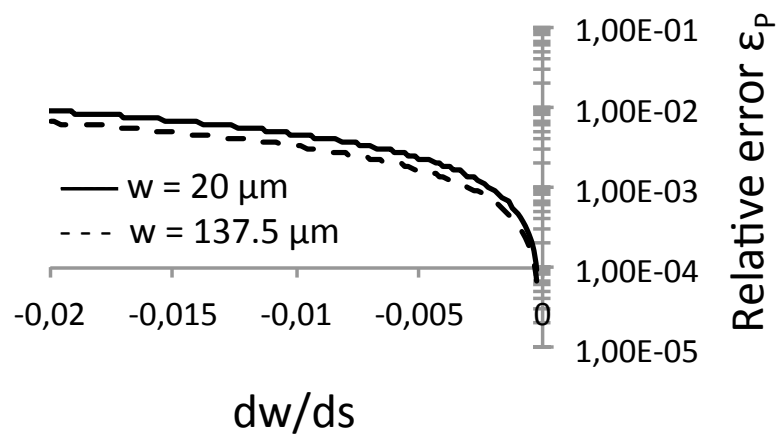

Fig. 4. Plot of the error in the approximative calculation of the capillary pressure $P(s) v s$ the slope of the channel $d w / d s$.

As can be seen in Fig. 3, the absolute slope of w(s) is very small throughout the channel, except for regions in the immediate vicinity of $s=L_{\max }$. Hence, the error on the estimation of $\mathrm{P}(\mathrm{s})$ remains negligible small as well. The maximum error occurs at $\mathrm{s}=$ $\mathrm{W}_{\text {min }}$, at which position the slope can be calculated using eq. 15 . For our design example, $\left.\frac{d w}{d s}\right|_{w=w_{\min }}=-9 \mathrm{e}-4$, and the error $\varepsilon_{P}<3.5 \mathrm{e}-3$.

The third assumption pertains to the approximative factor 0.630 in the calculation of the channel resistivity in eq. 9. The error on the channel resistivity introduced by this approximation can be estimated, by truncating the sum after the fourth term, as 3.7e-3 \pm $6 e-5$ for $w / h=0.5$. Hence, the deviation of the flow rate remains below this figure.

The above thus verifies that for a large range of CP designs, the three approximations will indeed have a negligible influence on the flow rate of the device.

Note that neglecting the higher order terms in the derivation of eqs. 9 and 11 are key to allowing the integration to obtain eq. 16 in parametric form.

Note also that the derivation of a single channel CP with circular cross-section can be derived in a similar fashion, but without the need for series approximations. 


\section{Multichannel and micropillar array CPs with constant flow rate}

This section deduces the design rules for multichannel CPs with a desirable pump volume $\mathrm{V}$ and a constant flow rate $\mathrm{Q}_{0}$, and using a technology that allows a maximum channel height $\mathrm{h}$, but under the restriction of a maximum channel length, $\mathrm{L}_{\mathrm{ch}}$. Consider a multichannel design, consisting of $n$ identical parallel channels featuring a shape $w_{n}(s)$ or $\mathrm{s}_{\mathrm{n}}(\mathrm{w})$, defined by eqs. 25 and 26 , that are each designed for a flow rate $\mathrm{Q}_{0} / \mathrm{n}$ and that are truncated at $\mathrm{s} \approx \mathrm{L}_{\mathrm{ch}} . \mathrm{w}_{\mathrm{n}}\left(\mathrm{L}_{\mathrm{ch}}\right)$ defines $\mathrm{w}_{\mathrm{n} \text {,min. }}$. One can choose the channel height and maximum width for each of these channels as $h=h_{\max }$ and $w_{\max }=h_{\max }$. Each of the parallel channels requires an upstream flow resistance $R_{n, 0}=n \cdot R_{0}$. However, due to the parallel nature of the design, the total upstream flow resistance equals $R_{n, 0} / n=R_{0}$, which is independent from $\mathrm{n}$. A suitable value for $\mathrm{n}$ can be determined by demanding that the volume of each channel,

$\mathrm{V}_{\mathrm{n}}=\mathrm{V} / \mathrm{n}$.

If $\mathrm{L}_{c h}<<\mathrm{L}_{\max }$ one can assume that

$\mathrm{W}_{\max }-\mathrm{W}_{\min }<<\mathrm{W}_{\max }$

in which case one can estimate

$V_{n} \approx w_{\max } \cdot h \cdot L_{c h}=\frac{h^{2} L_{c h}}{2}$.

Subsituting eq. 32 in 34 allows estimating

$n \approx \frac{2 . V}{h^{2} L_{c h}}$.

All design parameters are now unambiguously defined. When determining the design for a CP with geometrical design parameters and liquid properties as defined in Table 2, and demanding the same pump volume of $10.5 \mu \mathrm{l}$, but with $\mathrm{L}_{\mathrm{ch}}<2.5 \mathrm{~cm}$, one can use eq. 35 to estimate a minimum required amount of parallel channels $n=11$. For a perfect match to the required pump volume, the channels must be truncated at $x=2.52 \mathrm{~cm}$. At this position, $\mathrm{w}_{\min }=\mathrm{w}_{\mathrm{n}}(\mathrm{s}=2.52 \mathrm{~cm})=133 \mu \mathrm{m}$, validating the assumption in eq. 33 . Note that in this example, the design features a CP consisting of parallel channels with nearly constant rectangular cross-section. This is possible because the flow resistance of the CP $<<\mathrm{R}_{0}$, which was the a priori design requirement of previous CP designs (Eddowes, 1988; Juncker, 2002; Gervais, 2011; Mirzaei, 2010). 


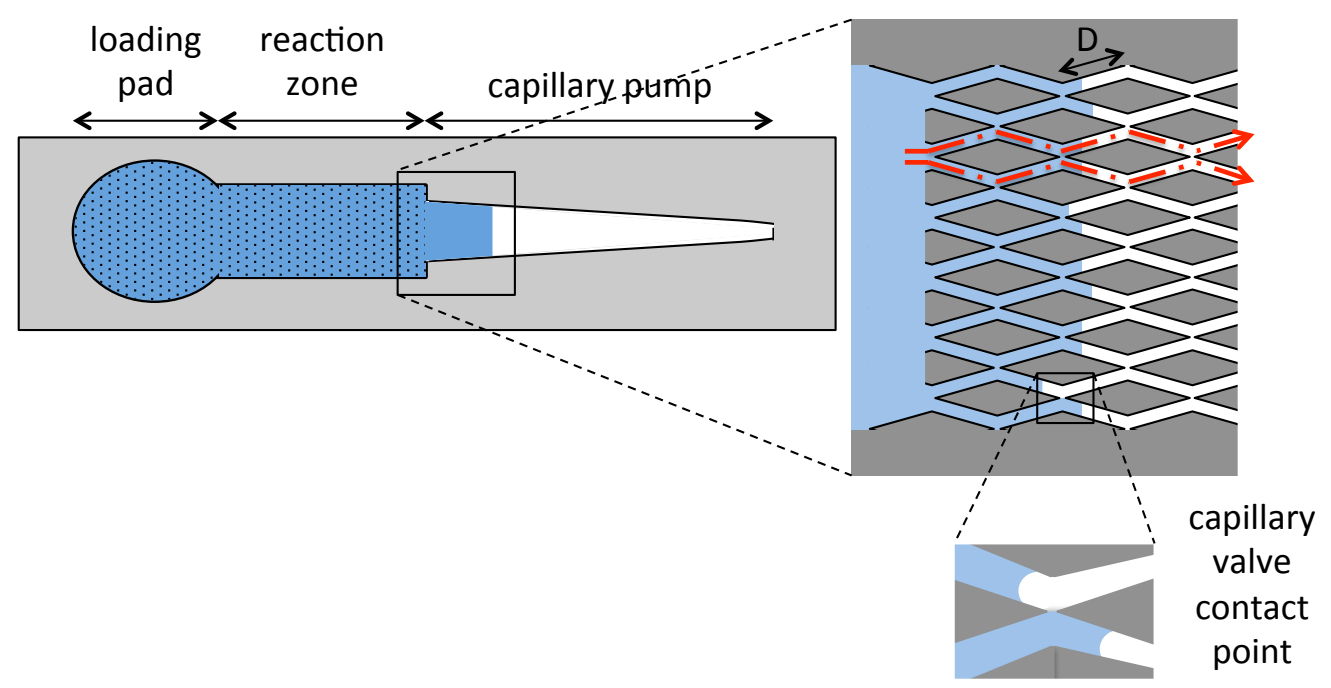

Fig. 5. Schematic of a micropillar array CP design with constant flow rate (left) with closeups of the pillar and geometric capillary valves openings between meighbouring channels (right). The red dash-dotted lines indicate the channel centre line for two of the parallel channels.

Capillary pumps are often designed as micropillar arrays, which, due to their large amount of fluidic interconnections, are inherently robust against channel blockage by debris and against manufacturing imperfections. The multichannel CP design derived above can be adapted into a micropillar array design by designing the parallel channels to contact their neighbour channels at periodic length positions D, as illustrated in Fig. 5. Each channel contact point can be designed as a narrow opening that functions as a geometric capillary valve (Melin, 2004) to avoid bubble trapping: if only one side of an opening is wetted, the surface energy prevents the liquid-gas interface to cross the opening. Once both sides of the opening are wetted, flow through the opening is allowed. The multiple inter-channel openings decrease differences in flow speed and pressure drop between adjacent channels.

\section{Capillary pumps for arbitrary, non-constant, flow rates}

To obtain a flow profile $Q(t)$ that is a multistep function in t, i.e. consisting of $n$ consecutive constant flow rates

$\mathrm{Q}(\mathrm{t})=\mathrm{Q}_{\mathrm{j}}$ for $\mathrm{t}_{\mathrm{j}}<\mathrm{t}<\mathrm{t}_{\mathrm{j}+1}$ and with $\frac{d Q_{j}}{d t}=0$, with $\mathrm{j}=1, \ldots \mathrm{n}$,

a CP can be designed as a concatenation of $n$ single channel CPs with constant height $h$ and with a general shape defined by eqs. 25 or 26, as illustrated in Fig. 6. 


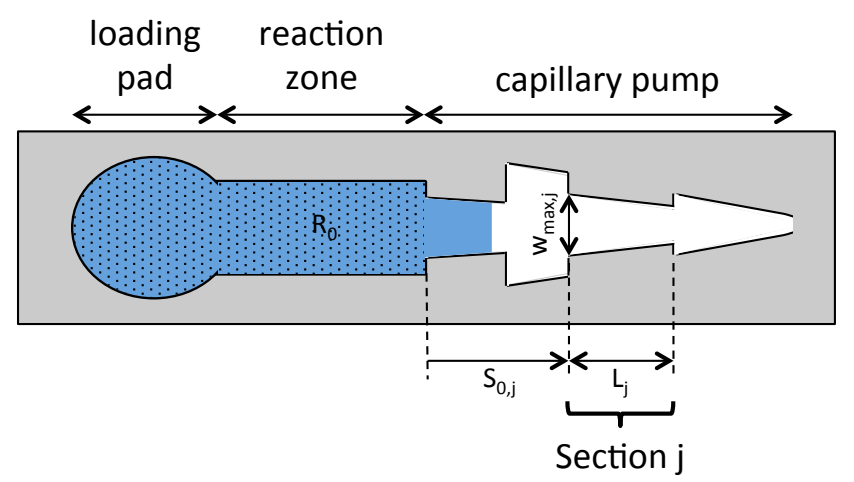

Fig. 6. Schematic of a CP design that provides a multistep function flow profile.

The shape $\mathrm{w}_{1}(\mathrm{~s})$ of the first section and the upstream flow resistance $\mathrm{R}_{0}$ can be calculated as described above. For the following channel sections, the shape $w_{j}(s)$ is entirely defined by the starting position $s=s_{0, j}$, the starting width, $w_{\max , j}=w_{j}\left(s_{0, j}\right)$ and the section length $\mathrm{L}_{\mathrm{j}}$. For consecutive sections $\mathrm{j}$, these three parameters can be defined as follows.

$s_{0, j}=\sum_{k=1}^{j-1} L_{k}$

$\mathrm{W}_{\max , \mathrm{j}}$ can be solved from

$\mathrm{P}\left(\mathrm{s}_{0, \mathrm{j}}\right)=2 \cdot \gamma \cdot \cos (\theta) \cdot\left[\frac{1}{h}+\frac{1}{w_{\max , j}}\right]$,

in which

$P\left(s_{0, j}\right)=Q_{j} \cdot R_{c h}\left(s_{0, j}\right)$,

with the total upstream flow resistance $\mathrm{R}_{\mathrm{ch}}\left(\mathrm{s}_{0, \mathrm{j}}\right)$ defined by eq. 8 .

The length $\mathrm{L}_{j}$ can be calculated from

$V_{j}\left(L_{j}\right)=Q_{j} \cdot\left(t_{j+1}-t_{j}\right)$,

in which $V_{j}$ is the volume of section $j$, which equals (eq. 21)

$V_{j}=\frac{\gamma \cdot \cos (\theta) \cdot h}{6 \cdot \mu \cdot Q_{j}} \cdot\left[\frac{0.21}{4}\left(w_{\max }{ }^{4}-L_{j}{ }^{4}\right)-\frac{h}{6} \cdot\left(w_{\max }{ }^{3}-L_{j}{ }^{3}\right)-w_{\max }{ }^{2} \cdot\left(0.21 \cdot w_{\max }-\frac{h}{2}\right) \cdot\left(w_{\max }-L_{j}\right)\right]$.

The concatenation of a section $\mathrm{j}$ for which $\mathrm{w}_{\mathrm{j}}\left(\mathrm{s}_{0, j}\right)<\mathrm{w}_{j-1}\left(\mathrm{~L}_{j-1}\right)$, i.e. if $\mathrm{Q}_{j}>\mathrm{Q}_{\mathrm{j}-1}$, is straightforward. However, when $w_{j}\left(S_{0, j}\right)>w_{j-1}\left(L_{j-1}\right)$, i.e. if $Q_{j}<Q_{j-1}$, care must be taken that the sudden expansion of the channel at the concatenation point does not create a passive capillary valve. This can be assured, e.g., by introducing a smooth channel expansion from channel positions $\mathrm{L}_{\mathrm{j}-1}$ to $\mathrm{s}_{0, \mathrm{j}}$ such that $\frac{1}{2}$. atan $\left(\frac{d w}{d s}\right)<\frac{\pi}{2}-\theta$, as described by Man et al. (Man, 1998). Designs where $w(s)$ becomes smaller than the manufacturable channel width can be replaced by multichannel designs in a similar fashion as outlined in the previous section.

The remainder of this section briefly discusses shape solutions for CPs for arbitrary, non-constant, flow profiles $Q(t)$. For every liquid front position $\mathrm{x}$, 
$Q(x)=A \cdot \bar{u}(x)=w \cdot h \cdot \frac{d x}{d t^{\prime}}$

where $\mathrm{A}$ is the flow cross-sectional area and $\bar{u}$ is the flow velocity of the liquid front. Equation 5 can be rewritten as

$R_{h}(x)=\frac{P(x)}{Q(t)}$,

which after differentiating in s results in

$\mathrm{R}(\mathrm{x})=\frac{1}{Q(t)} \cdot \frac{-2 \cdot \gamma \cdot \cos (\theta)}{w^{2}} \cdot \frac{d w(x)}{d x}-\frac{1}{Q^{2}(t)} \cdot \frac{\frac{d Q}{d t}}{\frac{d x}{d t}} \cdot P(x)$.

Substituting eqs. 7 and 42 in 43 and restructuring the terms leads to a first order differential equation in $\mathrm{w}(\mathrm{x})$ of the form

$\frac{\mathrm{T}_{1}(\mathrm{w})}{T_{2}(w)} \cdot \frac{d w}{d x}=1$,

with $\mathrm{T}_{1}(\mathrm{w})$ a $2^{\text {nd }}$ order polynomial in $\mathrm{w}$ and $\mathrm{T}_{2}(\mathrm{w})$ a $5^{\text {th }}$ order polynomial in $\mathrm{w}$. An algebraic expression for the solution of $w(x)$ is based on the roots of $T_{2}(w)$. Although there exists at least one real root of $\mathrm{T}_{2}$, the Abel-Ruffini theorem states that it is impossible to formulate a general parametric solution for the $\operatorname{root}(\mathrm{s})$. I.e., the $\operatorname{root}(\mathrm{s})$ is/are real, but cannot always be described in an algebraic form of radicals. However, some specific parameter choices may be algebraically solvable. Furthermore, it may be possible to solve this design challenge using numerical approximations. However, these challenges are not addressed in the scope of this paper.

\section{Conclusion}

This paper unambiguously defines the geometric parameters for constant flow rate capillary pumps, and derives the shape of such pumps in the form of a single channel, a multichannel array, and a micropillar array. Furthermore, the capillary pump shape for for a multistep constant flow profile is derived. Finally, it is shown that it is not possible to $a b$ initio derive an algebraic equation describing the shape of a capillary pump for an arbitrarily varying flow rate.

\section{Acknowledgements}

This work received partial financial support from the European Commission in the framework of the FP7 project ROUTINE. This manuscript was conceived and written at the border of Lake Särkijärvi in Finnish Lapland during July 2013, and I would like to thank my wife and children for their patience, Mumma for the cookies, and Ukki for the fish.

\section{References}

Bruus, H., "Theoretical Microfluidics” (Oxford University Press, 2008)

Chandra, D., "Capillary Force in High Aspect-Ratio Micropillar Arrays", PhD thesis, University of Pennsylvania, 2009.

Eddowes, R. M. J., " Controlled flow rate capillary suction pump" United Kingdom Patent GB2208324, 1988 
Gervais, L., Hitzbleck, M. \& Delamarche, E. "Capillary-driven multiparametric microfluidic chips for one-step immunoassays", Biosensors and Bioelectronics 27, 64-70 (2011). 85

Juncker, D. et al. "Autonomous microfluidic capillary system", Analytical Chemistry 74, 6139-6144 (2002).

P. F. Man, C. H. Mastrangelo, M. A. Burns, and D. T. Burke,"Microfabricated capillaritydriven stop valve and sample injector," Proc. IEEE MEMS'98 Conference, pg. 45-50, Heidelberg, Germany, Feb. 1998

R.K. Shah, A. London, "Laminar flow forced convection in ducts : a source book for compact heat exchanger analytical data. Supplement 1," pp. 198-199, 1978, ISBN0-12020051-1

Melin, J., Gimenéz, G., Roxhed, N., van der Wijngaart, W., Stemme, G., "A fast passive and planar liquid sample micromixer", Lab Chip. 2004 Jun;4(3):214-9.

Mirzaei, M. et al. "Microfluidic perfusion system for culturing and imaging yeast cell microarrays and rapidly exchanging media", Lab on a Chip 10, 2449-2457 (2010).

Safavieh and Juncker, D., "Serpentine and leading edge capillary pumps", Proc. microTAS 2011, Seatle, USA. 


\section{Appendix}

This appendix derives an expression for the capillary pressure in a channel with rectangular cross-section. We will first derive such expression for a zero-th order Taylor series approximation for $\mathrm{w}(\mathrm{s})$ around $\mathrm{s}=\mathrm{x}$, and thereafter for first order Taylor series approximation. The latter will allow estimating the maximum error induced by the zeroth order approximation, and hence the interval of $s$ where such approximation is valid. The below is an expansion of the work by (Man et al., 1998). We start from

$P(s)=\gamma \cdot\left[\cos (\theta) \frac{d A_{s l}}{d V_{l}}-\frac{d A_{l g}}{d V_{l}}\right]$,

where $U_{S}, V_{l}, A_{s l}, A_{l a}$ and $\gamma$ denote the total surface energy of the channel geometry, the liquid volume in the channel, the total wetted area of the channel, the total liquid-air interfacial surface area and the liquid-gas surface energy, respectively.

For a zero-th order approximation $\mathrm{w}(\mathrm{s})=\mathrm{w}(\mathrm{x})$, the capillary pressure equals that of a liquid in a rectangular channel with constant cross-section, see Fig. A1.

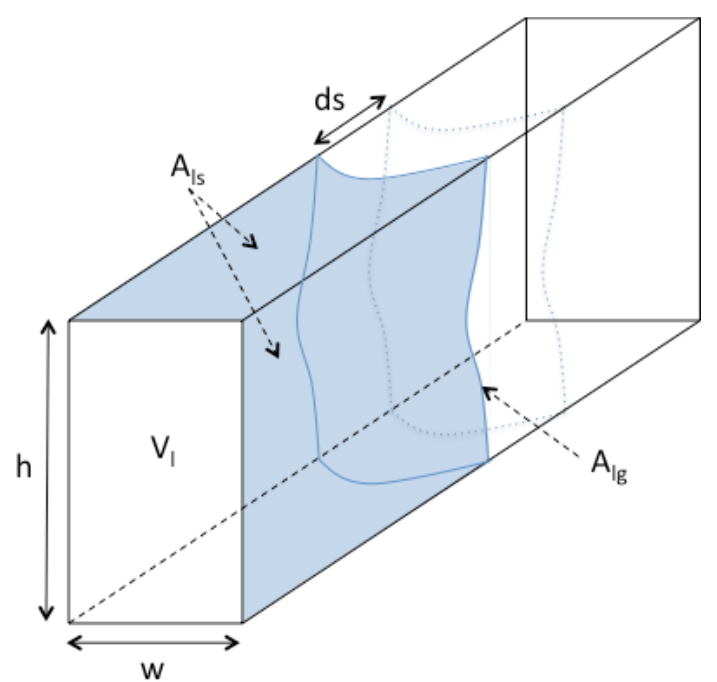

Fig. A1. Perspective illustration of the liquid in a capillary channel with constant rectangular cross-section.

The shape and area $A_{\lg }$ of the liquid-gas interface remains constant as the liquid front advances, and hence

$\frac{d A_{l g}}{d V_{l}}=0$.

$\frac{d A_{s l}}{d V_{l}}=\frac{\frac{d A_{s l}}{d s}}{\frac{d V_{l}}{s}}=\frac{\frac{(2 \cdot w+2 . h) \cdot d s}{d s}}{\frac{w \cdot h \cdot d s}{d s}}$.

Inserting eqs. $\mathrm{A} 2$ and $\mathrm{A} 3$ in $\mathrm{A} 1$ results in

$P_{0}(s)=2 \cdot \gamma \cdot \cos (\theta) \cdot\left[\frac{1}{h}+\frac{1}{w}\right]$.

For a first order approximation $\mathrm{w}(\mathrm{s})$ around $\mathrm{x}$, the capillary pressure equals that of a liquid in a tapered channel with a rectangular cross-section of which the width $\mathrm{w}$ decreases linearly with increasing s, as shown in top view in Fig. A2. 


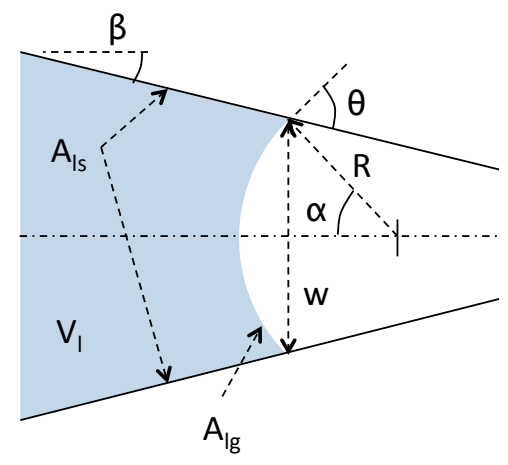

Fig. A2 Top view illustration of the capillary filling of a tapered channel, here illustrated as narrow slit channel with cylindrical liquid-gas surface.

As in the above case, the liquid-gas interface constitutes a complicated 3D surface that cannot be generally described parametrically. If $w<h$, the shape of the interface surface is close to that of a parallel plate slit, i.e. cylindrical. We will here solve the capillary pressure drop for such cylindrical surface shape, as depicted in Fig. A2, and assume that the results can be extended to general liquid surface geometries for $w \leq h$. Defining

$\beta=\frac{1}{2} \operatorname{atan}\left(\frac{d w(x)}{d s}\right)$

$\alpha=\pi / 2-\theta-\beta$,

and

$R=\frac{w}{2 \cdot \sin \alpha}$

we can calculate

$d V=-h \cdot\left[\frac{1}{\operatorname{tg} \beta}-\frac{1}{\operatorname{tg} \alpha}+\frac{\alpha}{\sin ^{2} \alpha}\right] \cdot \frac{w}{2} \cdot \frac{d w}{d s} \cdot d s$,

$d A_{l g}=2 \alpha h d R=\frac{\alpha h}{\sin \alpha} \cdot \frac{d w}{d s} \cdot d s$, and

$d A_{s l}=-\left[\frac{2 \cdot h}{\cos \beta}+2 \cdot\left[\frac{1}{\operatorname{tg} \beta}-\frac{1}{\operatorname{tg} \alpha}+\frac{\alpha}{\sin ^{2} \alpha}\right] \cdot \frac{w}{2} \cdot \frac{d w}{d s} \cdot\right] \cdot d s$.

Substituting A5, A7, A8, and A9 in A1 results in

$P_{1}(s)=\gamma \cdot\left[\cos \theta \cdot \frac{2}{h}+\frac{\frac{2 \cdot h}{\cos \beta} \cdot \cos \theta}{h \cdot\left[\frac{1}{\operatorname{tg} \beta}-\frac{1}{\operatorname{tg} \alpha}+\frac{\alpha}{2 \cdot \sin ^{2} \alpha}\right] \cdot w \cdot \frac{d w}{d s}}+\frac{\frac{\alpha h}{\sin \alpha} \cdot \frac{d w}{d s}}{h \cdot\left[\frac{1}{\operatorname{tg} \beta}-\frac{1}{\operatorname{tg\alpha } \alpha}+\frac{\alpha}{2 \cdot \sin ^{2} \alpha}\right] \cdot \frac{d w}{d s} \cdot w}\right]$,

which can be rewritten as

$P_{1}(s)=\gamma \cdot\left[\frac{2 \cdot \cos \theta}{h}+\frac{2 \cdot \cos \theta}{w} \cdot \frac{2}{\cos \beta \cdot\left[\frac{1}{\operatorname{tg} \beta}-\frac{1}{\operatorname{tg} \alpha}+\frac{\alpha}{\sin ^{2} \alpha}\right] \cdot \operatorname{tg}(2 \beta)}+\frac{2 \cdot \alpha}{\left[\frac{\sin \alpha}{\operatorname{tg} \beta}-\cos \alpha+\frac{\alpha}{\sin \alpha}\right] \cdot w}\right]$. 